\title{
New Procedure of Biocompatibility Increment of Transparent Materials via Picosecond Pulse lonization Process
}

\author{
Naghmeh Safaie, Amirkianoosh Kiani \\ Silicon Hall: Laser Micro/Nano Fabrication Facility, Faculty of Engineering and Applied Science, Ontario \\ Tech. University (UOIT) \\ Oshawa, ON, Canada \\ amirkianoosh.kiani@uoit.ca
}

\section{Extended Abstract}

Generally, transparent materials such as glass and bio-glass have extensive applications in tissue engineering; researchers have gone through many investigations to make them more compatible with body organs. The high intensity laser induced reverse transfer method (HILIRT) is a simple and quick plasma ionization method for enhancement of biocompatibility of transparent biomaterials via generation of a thin-film of nano fibrous Ti (NFTi) structures on the glass [1].

In this study, nanofibrous titanium on glass substrate have been prepared by a chemical-free process called high intensity laser induced reverse transfer method (HILIRT), and followed by introducing an analytical model for evaluation of synthesized structures via picosecond to nanosecond laser pulses. The high-energy laser pulses are directed and centralized to a very small point on titanium sheet, which pass through the transparent glass (gap between glass and Ti target: $500 \mathrm{um}$ ) in a very short time ranging from 150 picosecond to 30 nanoseconds. Transferred laser energy leads to the alteration and ablation and finally deposition of different titanium nano/micro structures on the glass surface.

In this research, titanium nanofibers with different structural properties on simple transparent microscope slides were synthesized using different laser pulse durations. The morphology of coated nanostructured titanium on glass substrate were analyzed by scanning electron microscopy (SEM), while simulation ablation process was conducted by coding in MATLAB R2015b software. The SEM results displayed that diminishing laser pulse duration from nanosecond to picosecond leads to the more titanium nanofibers growth on the glass substrate. Additionally, higher-temperature and deeper heated affected zone (HAZ) has been observed in theoretical results for shorter pulse duration of $150 \mathrm{ps}$ which is in good agreements with experimental results $[2,3]$. The X-Ray Diffraction (XRD) results of the fabricated NFTi structures show higher peaks of titanium (alpha) and titanium oxide phases on samples treated at shorter laser pulse duration of $150 \mathrm{ps}$. It can be the outcome of a forming of higher temperature and denser plasma plume at shorter pulse duration that contains more particles and ions of titanium, which leads to generation of thicker and denser NFTi thin-film on the glass substrate [4].

\section{References}

[1] N. Safaie, A. Kiani, "Enhancement of bioactivity of glass by deposition of nanofibrous Ti using high intensity laser induced reverse transfer method," Vacuum., vol. 157, pp. 92-99, 2018.

[2] E. Akman, A. Demir, T. Canel, T. Sınmazçelik, "Laser welding of Ti6Al4V titanium alloys," J. Mater. Process. Tech., vol. 209, no. 8, pp. 3705-3713, 2009.

[3] S. T. Hendow, S. A. Shakir, "Structuring materials with nanosecond laser pulses," Opt .Express, vol. 18, no. 10, pp. 10188-10199, 2010.

[4] C. Colpitis, A. Kiani, "Synthesis of bioactive three-dimensional silicon-oxide nanofibrous structures on the silicon substrate for bionic devices' fabrication," Nanomater. Nanotechno, vol. 6, no. 8, pp. 1-7, 2016. 